ISSN 2079-9292

www.mdpi.com/journal/electronics

Article

\title{
Modified EAS Tag Used as a Resistive Sensor Platform
}

\section{Henrik Andersson ${ }^{1}{ }^{1}$, Krister Hammarling ${ }^{1}$, Johan Sidén ${ }^{1}$, Anatoliy Manuilskiy ${ }^{1}$, Thomas Öhlund ${ }^{2}$ and Hans-Erik Nilsson ${ }^{1}$}

1 Electronics Design Division, Department of Information Technology and Media, Mid-Sweden University, SE-851 70, Sundsvall, Sweden; E-Mails: Krister.Hammarling@miun.se (K.H.); Johan.Siden@miun.se (J.S.); Anatoliy.Manuilskiy@miun.se (A.M.); Hans-Erik.Nilsson@miun.se (H.-E.N.)

2 Digital Printing Center, Department of Applied Science and Design, SE-891 18, Örnsköldsvik, Sweden; E-Mail: Thomas.Ohlund@miun.se

* Author to whom correspondence should be addressed; E-Mail: Henrik.Andersson@miun.se; Tel.: +46-70-3622245; Fax: +46-60-148456.

Received: 6 September2012; in revised form: 30 October 2012 / Accepted: 30 October 2012 / Published: 9 November 2012

Abstract: In this article, a modified design of an RF Radio Frequency Electronic Article Surveillance (EAS) tag, used as a sensor platform, is manufactured and characterized. EAS tags are passive devices consisting of a capacitor and coil, tuned to a resonance frequency readable by the detector equipment, in this case $8.2 \mathrm{MHz}$. They were originally used to detect whether merchandise was being moved through the detection gates at shop exits, in which case an alarm was triggered. If the capacitance is divided in two and a resistive sensor device inserted in between, the resonant Inductor-Capacitor (LC) circuit becomes an Inductor-Capacitor-Capacitor-Resistor LCCR circuit and can be used as a sensor tag. A high sensor resistance means that one capacitor is decoupled, leading to one resonance frequency, while a low resistance will couple both capacitances into the circuit, resulting in a lower resonance frequency. Different types of resistive sensors exist that are able to detect properties such as pressure, moisture, light and temperature. The tag is manufactured in Aluminum foil on a polyetylentereftalat (PET) substrate, resulting in a cost effective RF-platform for various resistive sensors. Two types of tags are designed and manufactured, one with parallel plate capacitors and the other with interdigital capacitors. To test the tags, a resistive tilt sensor is mounted and the tags are characterized using a network analyzer. It is shown that for high resistance, the tags have a resonance frequency 
of morethan $10 \mathrm{MHz}$ while for low values the frequency approaches $8.2 \mathrm{MHz}$.

Keywords: resonance circuit; RF-tag; EAS; surveillance

\section{Introduction}

Currently, the possibility to manufacture completely printed Radio Frequency Identification (RFID) tags is limited, mostly because of problems associated with printing high frequency transistors, usually in the MHz range. To date, many papers presenting the development of printed technology for RFID applications, including printed antennas and transistors, have been presented [1-14]. Research has previously been done on sensing applications using RFID technology [15,16]. A specific example is an article that shows how to produce an RFID tag that senses temperature by using a Shaped Memory Alloy (SMA). It is a part of an antenna that will change the impedance matching of an RFID chip so that it will be matched or mismatched depending on the temperature and thereby be readable or not. The tag also contains a second RFID chip that is unaffected by temperature changes so that the tag can always be detected as present [17].

However, it is still the case that the cost for implementing sensor applications using RFID tags can be too high in cost sensitive areas.

As an alternative, adding new functionality to a device consisting only of printed passive components opens up potential application areas in cost sensitive markets. Such a device is the Radio Frequency (RF) Electronic Article Surveillance (EAS) tag, traditionally used to detect whether merchandise has moved through detection gates at shop exits, in which case an alarm is triggered. They are based on a simple LC resonance circuit consisting of a capacitor and coil tuned to the detection frequency of the readout equipment. The reader generates an alternating magnetic field that induces energy in the EAS tag if it is located in the vicinity. If the frequency of the magnetic field and resonance frequency of the EAS tag match then this leads to a small but detectable voltage drop in the generating coil. In addition, an extra optional sensor coil can be used which senses a voltage drop when the tag is within the magnetic field. The damping resistance of the tag should be as low as possible, preferable with a quality factor (Q-factor) of $>60$ [18].

EAS tags are limited in their information capacity, just being able to be detected in the interrogation zone or not, hence also called 1-bit transponders, as compared to RFID chips that are commonly capable of communicating data in the order of kbits. The EAS tags are in the "on" state when manufactured and are deactivated in the shop by applying a strong magnetic field that induces a sufficiently high voltage to short circuit the plate capacitor. This is assisted by having pre-fabricated weakened points, so-called "dimples" [18].

This article discusses the manufacturing and characterization of a modified type of RF EAS tag meant to be used as a sensor platform for resistive sensors and that is detectable at two distinctly different frequencies depending on the sensor state.

It can be used in, for example, shipping, in combination with different sensors, where each container or box may have its own sensor. It then becomes possible to pick out the specific container/boxes, for which a "trigger event" has occurred. This technology can be used as a 
complement to perform standard quality checks in several areas such as transport, food and packaging [19-23].

It has previously been proven how the detection of certain events can be applied to silicon based UHF RFID solutions by letting sensor switching mechanisms decide whether an RFID tag should respond with its ID number or not, i.e., if the tag is able to operate or not. Switching an RFID tag on or off by connecting different parts of an RFID tag's antenna structure is for example shown in [16] wherein a mercury-based accelerometer switch tells about an items movement status, and in the work presented in [17] wherein shape-memory alloys are used for detection of thermal thresholds.

The design of the EAS tag in this work is based on the standard Inductor-Capacitor (LC) resonance circuit but is modified to include a second capacitor connected, in parallel, through a resistive sensor resulting in an Inductor-Capacitor-Capacitor-Resistor LCCR circuit. The electrical schematic layout of the modified EAS tag is shown in Figure 1, where the sensor is represented by a resistor element, denoted $\mathrm{R} 1$, the inductance by $\mathrm{L} 1$, and the capacitors by $\mathrm{C} 1$ and $\mathrm{C} 2$ respectively.

This means that the sensor placed between the capacitances, R1, will act as a resistive divider that couples capacitor $\mathrm{C} 2$ into the resonance circuit, consisting of $\mathrm{L} 1$ and $\mathrm{C} 1$, in proportion to its resistance.

When the resistance of the sensor is high, the extra capacitor $\mathrm{C} 2$ is basically disconnected from the circuit, giving a resonance frequency that corresponds only to L1 and C1.

When the sensor resistance is, instead, low, the extra capacitor $\mathrm{C} 2$ is connected in parallel with the first, resulting in a lowering of the resonance frequency.

In this manner it is possible to measure at one frequency range to determine whether the tags are present and working, and have another "alarm" or "trigger" frequency range to check that the sensor has been activated.

By adjusting the values of $\mathrm{C} 1$ and $\mathrm{C} 2$ to reach the two desired resonance frequencies it is also possible to design a tag, which uses a sensor that goes to high resistance and decouples $\mathrm{C} 2$ when an event happens.

The readout equipment can be based on the standard EAS tag alarm with the addition of detection at two frequency ranges. The possibility could also be considered to use a handheld reading unit for localizing one specific activated EAS sensor tag amongst many nearby tags.

Figure 1. Electrical schematic of the Inductor-Capacitor-Capacitor-Resistor LCCR tag.

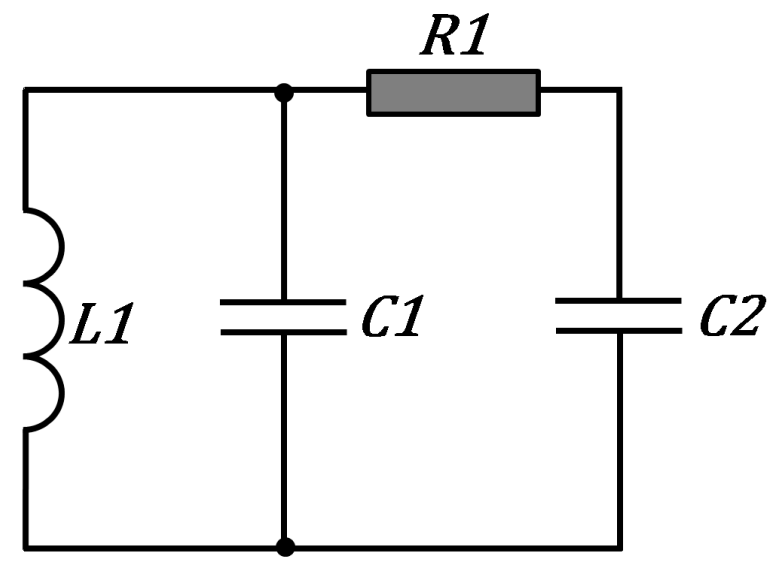


Different types of resistive sensors exist that are able to detect properties such as pressure, moisture, light, temperature and more. The requirement is that the resistance will be sufficiently low to activate the tag when the desired threshold of the measured quantity is reached.

For example, a tilt sensor could be mounted to check that packages are aligned correctly during transport. Some products are inherently sensitive to position during freight and should be transported in an upright, vertical direction in order to greatly enhance shock resistance. Reading this information electronically from the EAS tag provides the possibility of triggering an alarm if the packages are placed incorrectly and provides a better safety check as opposed to conducting only a visual inspection.

A latching acceleration switch (the switch sticks to its triggered state once the trigger event has occurred) could be mounted to check whether a package or container has been subject to shock during its previous transport. Products with an activated tag could be removed for further inspection avoiding the possibility of damaged products reaching a customer. Such impact-monitoring sensor can be built in a small size and will have no power requirements, as described in [24].

\section{Design and Simulation of LCCR EAS Tag}

The tags were manufactured in $50 \mu \mathrm{m}$ thick aluminum on a polyetylentereftalat (PET) substrate which is a manufacturing procedure that could provide a sufficiently low resistance in order to obtain an acceptable Q-factor. Tests show that a Q-factor of $\sim 30$, which corresponds to a series resistance of $6 \Omega$, is sufficient to trigger the EAS reader. However, this is the limit and includes the complete series resistance, with coil, capacitors and sensor.

Tests using ink-jet and screen printing techniques to apply conductive silver ink resulted in a resistance which was too high. Considering the relatively thin layer thickness obtained by ink-jet printing, usually in the range of $1 \mu \mathrm{m}$, it is deemed very difficult to produce sufficiently thick layers in order to obtain a low enough Q-value of a coil if the width of the lines is to be reasonable. Similar results are reported in [25] where coils are repeatedly screen printed up to 40 times so as to achieve a thickness of $350 \mu \mathrm{m}$ - but still only reach a Q-factor of 45 , and that with the extra cost of achieving non flexible prints.

The problem is apparent if considering the possible limit of the conductance for silver nano-particle ink, namely the same as bulk silver, that has a resistivity of $1.55 \times 10^{-8} \Omega \mathrm{m}$. Considering the geometry of the manufactured coil, which is $110 \mathrm{~cm}$ long and $1 \mathrm{~mm}$ wide, a $1 \mu \mathrm{m}$ layer of bulk silver would still result in a series resistance of $17.5 \Omega$, which gives a Q-factor of $\sim 11$, even when all the other serial resistance components are neglected [26].

This value is not reached for printed nano-silver ink structures even if using effective sintering methods such as electrical sintering and at best, possibly $50 \%$ of bulk silver conductance may be achievable [26-32]. The conclusion regarding ink-jet as a manufacturing method is that, at present, it is not feasible to manufacture coils with a line width in the range of $1 \mathrm{~mm}$ because of the limited layer thickness it can produce.

To predict the behavior of the LCCR tag, simulations were performed in order to estimate the frequency shift that different sensor resistance values will provide. In Figure 2, the schematic circuit layout used for the simulation is shown. 
Figure 2. Electrical layout used for the simulation of the Electronic Article Surveillance (EAS) tag reader and EAS tag.

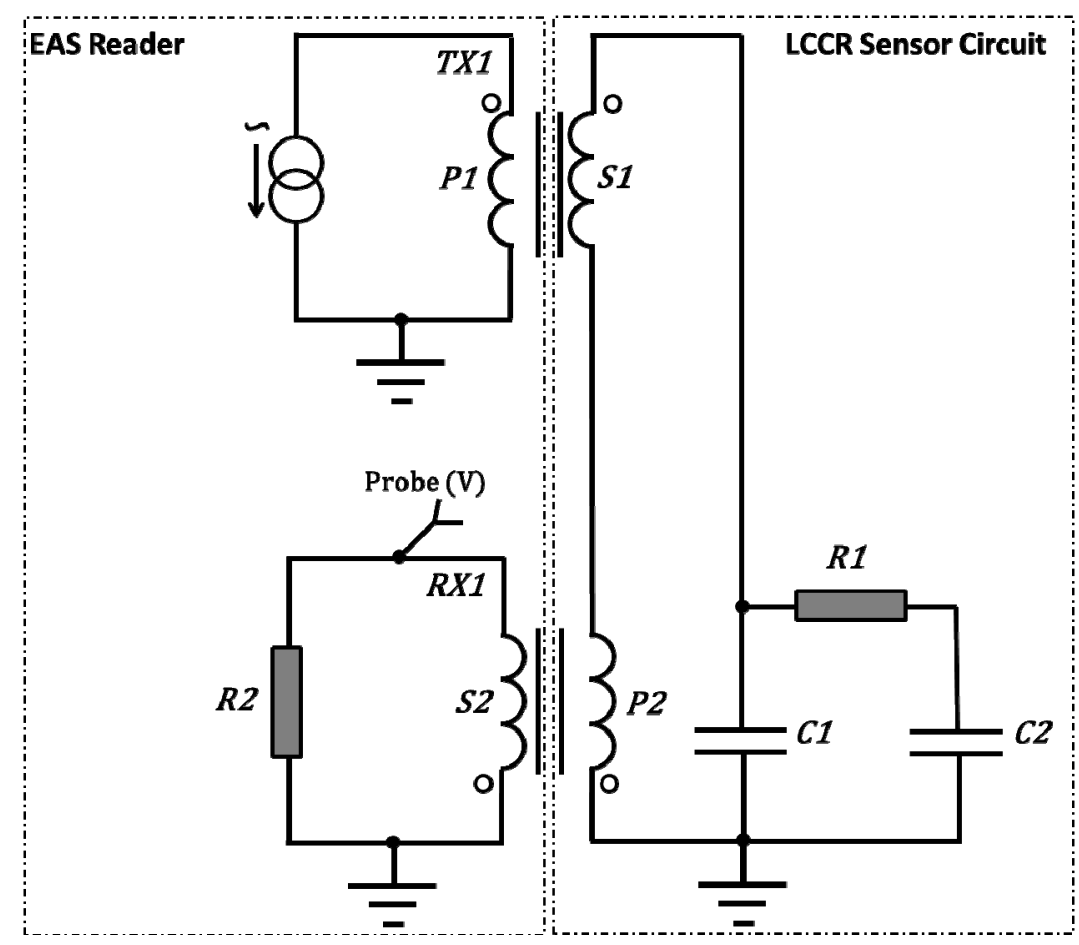

The simulation is setup so as to resemble the send/read gate and the tag in an EAS system. The sender coil (TX1) transfers energy to the LCCR circuit. The LCCR circuit then transmits its response to the readout coil (RX1). On the readout coil the voltage induced by the LCCR-circuit at different frequencies is probed. In the LCCR circuit part, resistor R1 acts as the sensor element, with low to high resistance values (on to off, respectively). The coupling efficiency of the transformers used has been set to 0.01 , i.e., only $1 \%$ of the energy is transferred from one part to the next in the system.

When the resistance becomes low, the equivalent capacitance moves towards $100 \mathrm{pF}$ and when the resistance is high the equivalent capacitance moves towards $60 \mathrm{pF}$. Figure 3 shows the simulated resonance frequency and the relative amplitude for resistance values between $1 \Omega$ and $100 \mathrm{k} \Omega$. It can be seen that the resonance frequency is $8.2 \mathrm{MHz}$ for 1 to $10 \Omega$ and about $10 \mathrm{MHz}$ for $10 \mathrm{k} \Omega$ and $100 \mathrm{k} \Omega$ and intermediate resistance values result in lower amplitudes and frequencies which fall in between.

This means that a sensor resistance of $100 \Omega$ or less provides the correct frequency to trigger the EAS detector used and values around some $\mathrm{k} \Omega$ or more offers a resonance peak around $10 \mathrm{MHz}$. It can also be observed that the damping in the circuit will be quite high for intermediate resistance values, therefore it is not necessarily true that the readout will trigger, even if the resonance frequency is correct, because the damping is too large. It is important to use sensors that have as low a resistance as possible when the tag is meant to trigger the EAS detector.

For the readout equipment targeted to be used in this work, manufactured by Gunnebo Nordic, the tag's resonance frequency should be $8.2 \mathrm{MHz}$, which is why the values chosen for the tag circuit shown in Figure 1 are: $\mathrm{L}=3.7 \mathrm{mH}, \mathrm{C} 1=60 \mathrm{pF}$ and $\mathrm{C} 2=40 \mathrm{pF}$ resulting in a frequency shift from about $10.5 \mathrm{MHz}$ with high resistance and $8.2 \mathrm{MHz}$ with low resistance. Smaller frequency shifts is possible by changing the values of the capacitors. 
Figure 3. Simulation results showing resonance frequency shift for different resistance values of the sensor.

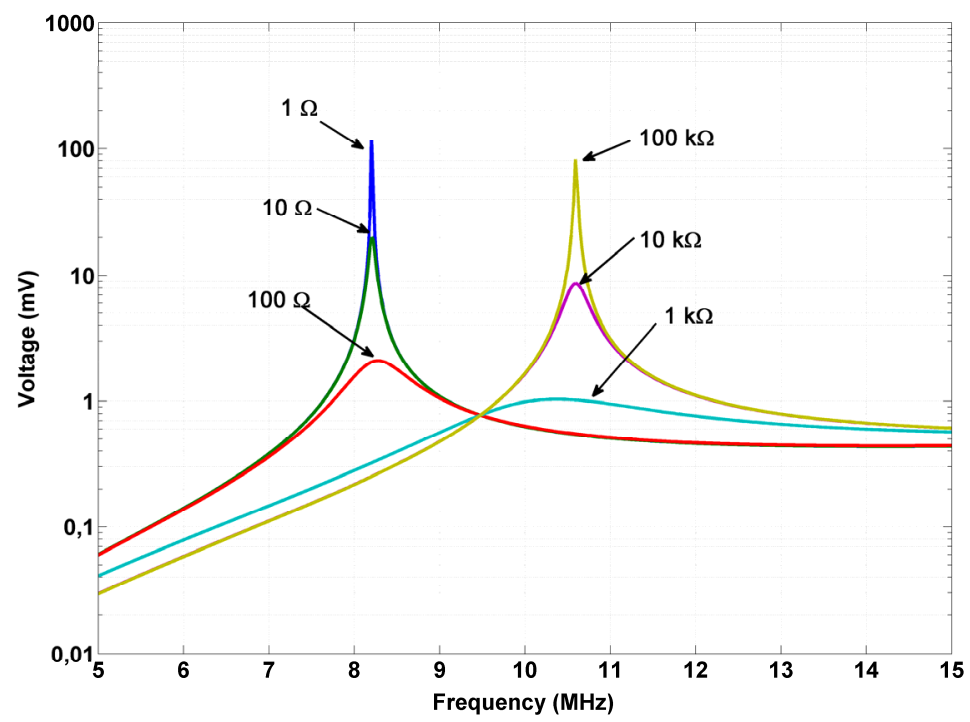

\section{Experimental Section}

The LCCR EAS tags have been manufactured in two different versions, one using parallel plate capacitors, and one which uses interdigital capacitors. Both tag types are constructed from $50 \mu \mathrm{m}$ thick aluminum foil on a $50 \mu \mathrm{m}$ PET substrate and the design was manufactured by the company Mekoprint (Denmark). In Figure 4, the aluminum foil layouts, the dimensions and sensor placement, and the positions of the probe of the network analyzer are shown. In the case of the parallel plate EAS tag, the coil was cut in the place indicated in Figure 4; in the case of the interdigital tag, the measurement was performed between the inner part of the coil and the capacitor before they were joined.

Figure 4. Top layer layout of the parallel plate capacitor LCCR EAS tag (left) and interdigital version (right) showing dimensions and the placement of the sensor.

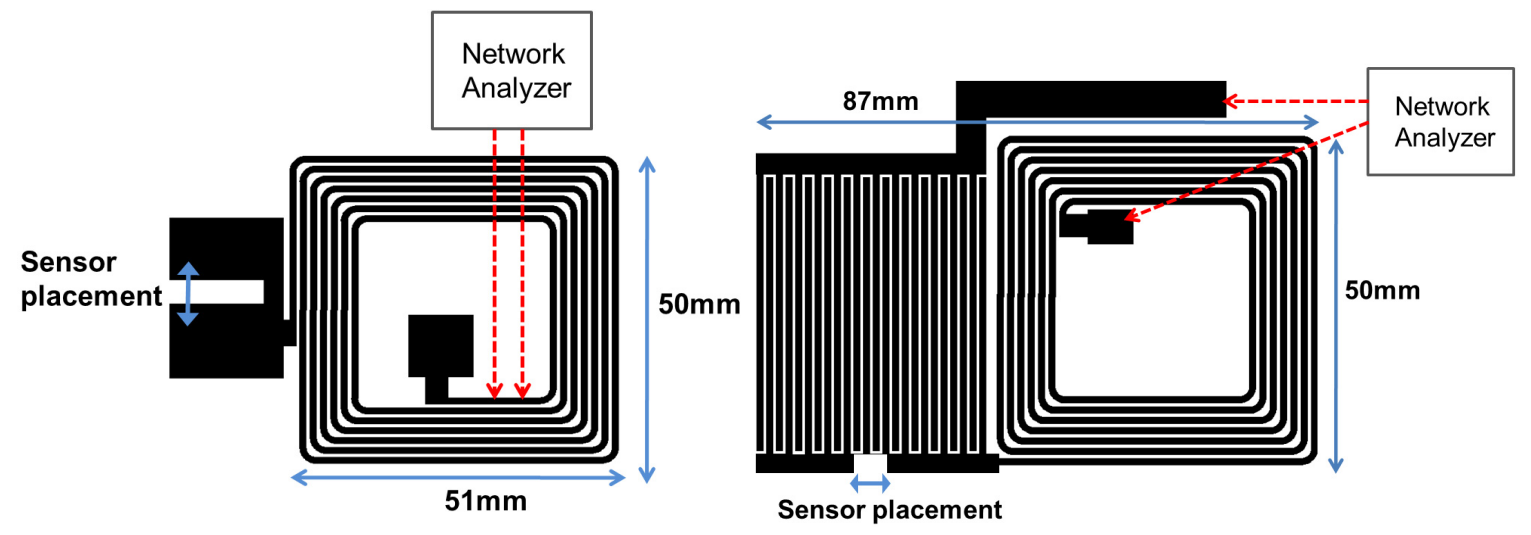

The parallel plate capacitor model utilizes the PET foil as a dielectric and a silver screen paste on the reverse side is used for the connection of the coil in addition to one plate of the capacitors, this can be seen in Figures 5 and 6. The connection between the inner part of the coil and the capacitors is achieved by removing the PET foil over the center contact by means of a Versa laser infrared laser 
cutting machine, thereby providing a via for the paste on the reverse side to connect to the aluminum on the front side. This can be seen in the right photo in Figure 5 as a square area in the middle of the coil.

Figure 5. Photos of plate LCCR EAS tags manufactured in aluminum on PET with tilt sensor, frontside, left and reverse side with printed screen paste, right.

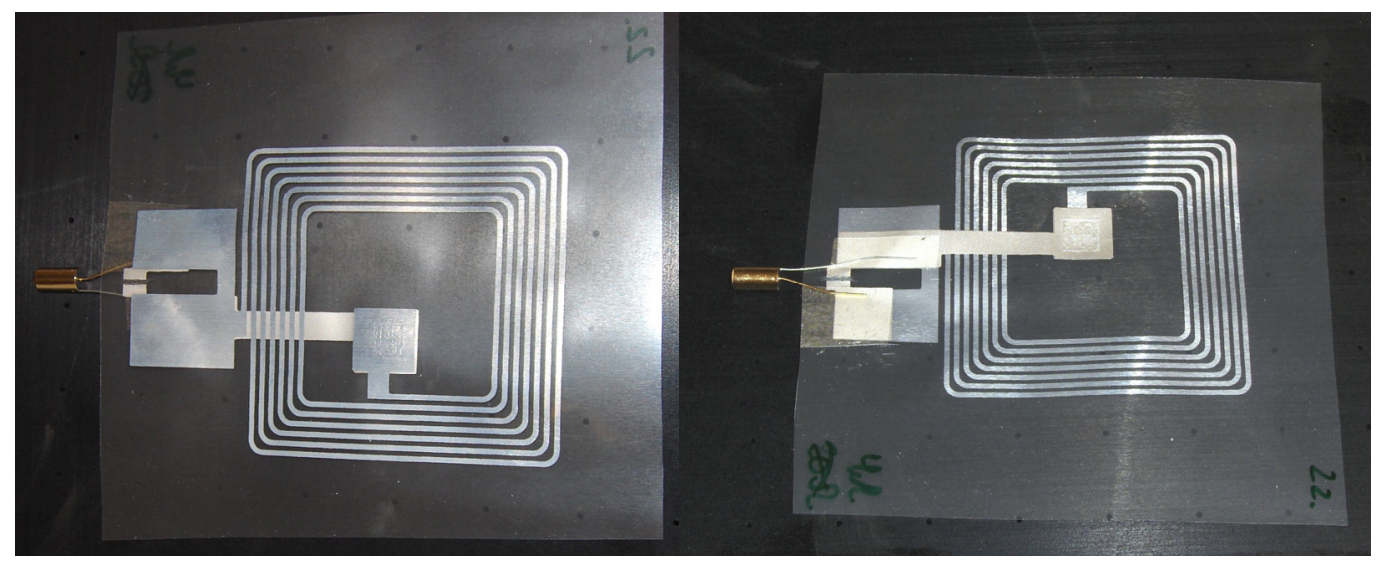

Figure 6. Photos of interdigital LCCR EAS tags manufactured in aluminum on PET with tilt sensor, frontside, left and reverse side with printed screen paste, right.

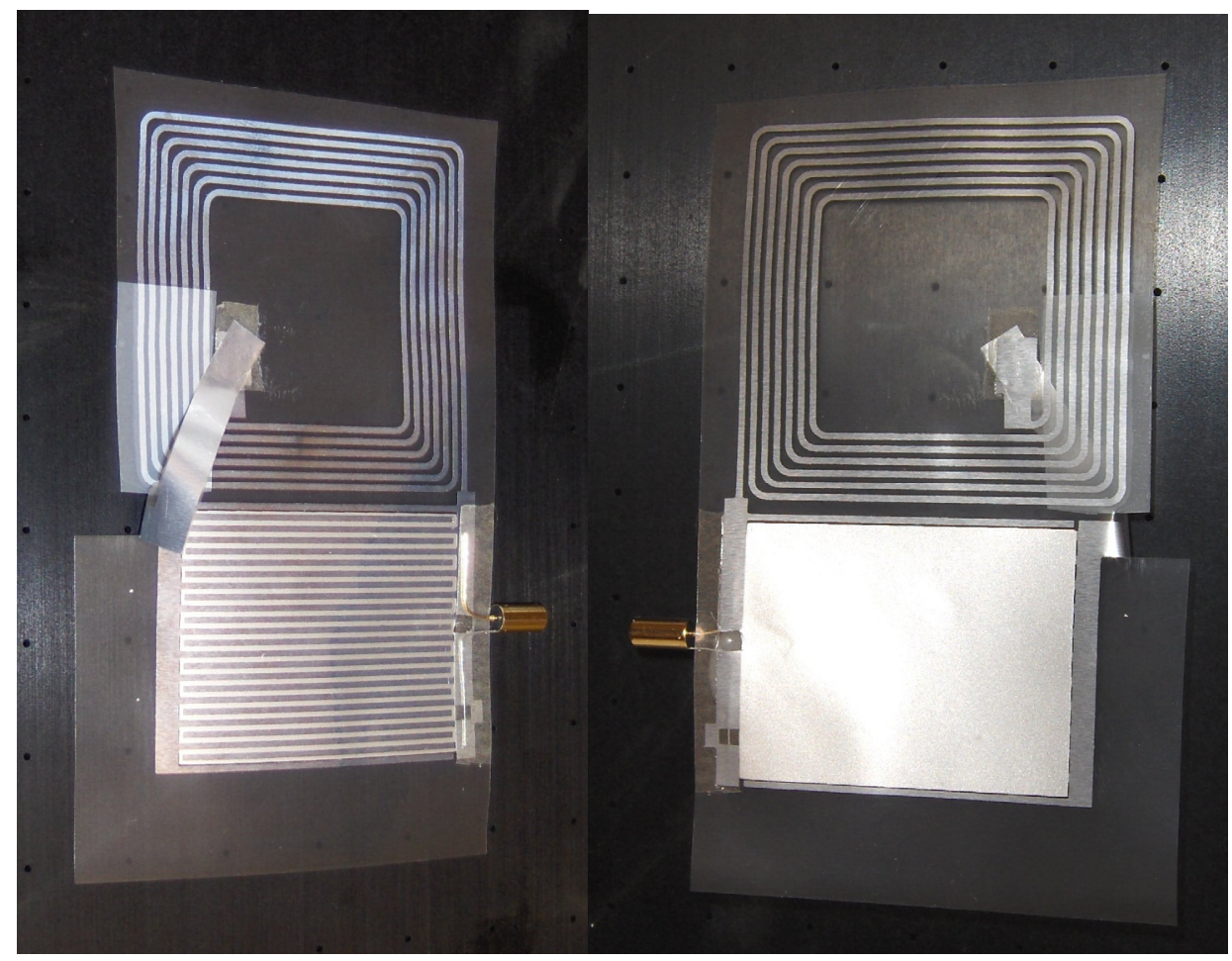

For the parallel plate EAS tag, the size of the capacitances is easily tuned by changing the area covered by the silver paste on the reverse side. The screen print of the silver paste on the reverse side was performed by the company Sweprod (Sweden) and can be seen in the photos in Figure 5.

For the interdigital model, the connection from the inner part of the coil to the capacitances is achieved by folding a piece of the foil and gluing it to the middle contact with anisotropic conductive tape, model 9705 manufactured by 3M (USA). This connection could also be achieved by, for example, silver epoxy or by other conductive glues or tapes. The capacitances are, in this case, also 
tuned by printing the silver screen paste on the reverse side of the interdigital structure, which will increase the capacitance in proportion to the area that is covered. Photographs showing the front and reverse sides of the plate capacitor model are shown in Figure 5 and the interdigital model is shown in Figure 6.

To demonstrate the function of the tags, a tilt sensor was mounted on both types, which can be seen in Figures 5 and 6. This was performed by first cutting a gap with scissors in the Al/PET foil and then attaching the sensors with anisotropic conductive tape $(3 \mathrm{M})$ at the positions indicated in Figure 4. An adjustment of the sensor position, as indicated in Figure 4, on the interdigital EAS tag will change the ratio of the capacitance that are coupled by the sensor thereby changing the resonance frequency in the un-triggered state.

The tilt sensor is a momentary rolling ball switch tilt sensor, model AT411-2 manufactured by the Light Country (China). The sensor works by having two metal balls inside the canister that close the circuit when it is upright, but, when tilted more than $30^{\circ}$, the balls will not connect with the contacts thereby creating an open circuit. The advantage of such a sensor type is that it is able to go from a very low resistance, $<1 \Omega$, to infinity, which suits the EAS sensor tag concept very well.

\section{Results and Discussion}

Measurements conducted using an Agilent E5070B network analyzer show that the resonance frequencies of the tags are very close to the simulated values.

Figures 7 and 8 show the measured impedance locus for the two EAS tag types. The markers show the change in resonance frequency when the mounted tilt sensor is in its high resistance "off" state compared to the low resistance "on" state. The resulting shift in resonance frequency is from $10 \mathrm{MHz}$ to $8.2 \mathrm{MHz}$ in the "on" state.

Figure 7. Smith chart showing measured resonance frequency for the interdigital EAS tag equipped with a tilt sensor that is in "on" state, blue (solid) graph, and "off" state, red (dashed) graph.

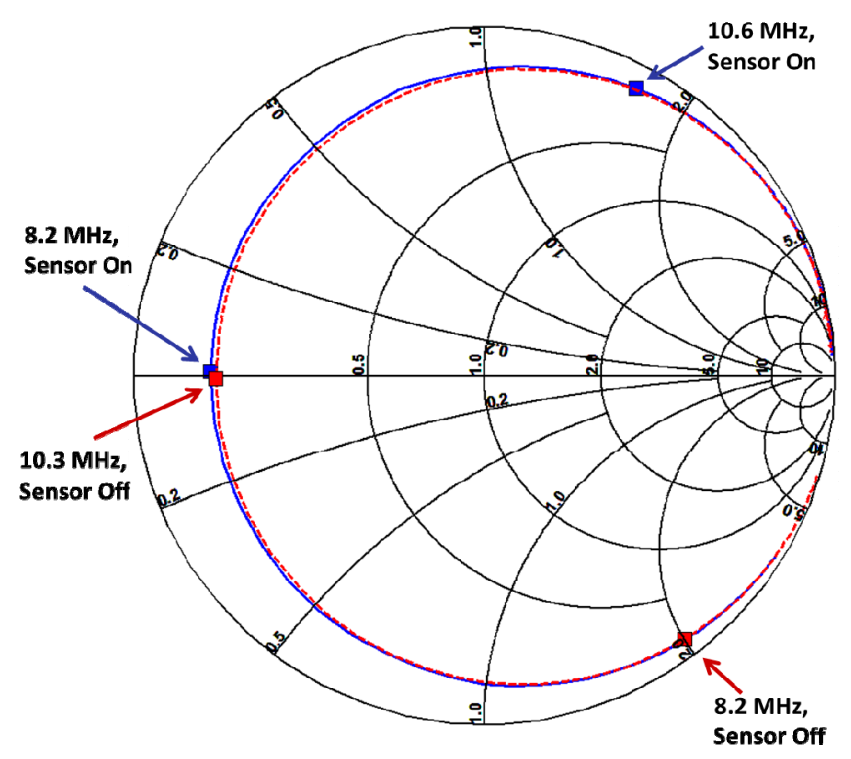


Figure 8. Smith chart showing measured resonance frequency for the parallel plate type EAS tag equipped with a tilt sensor that is in "on" state, blue (solid) graph, and "off" state, red (dashed) graph.

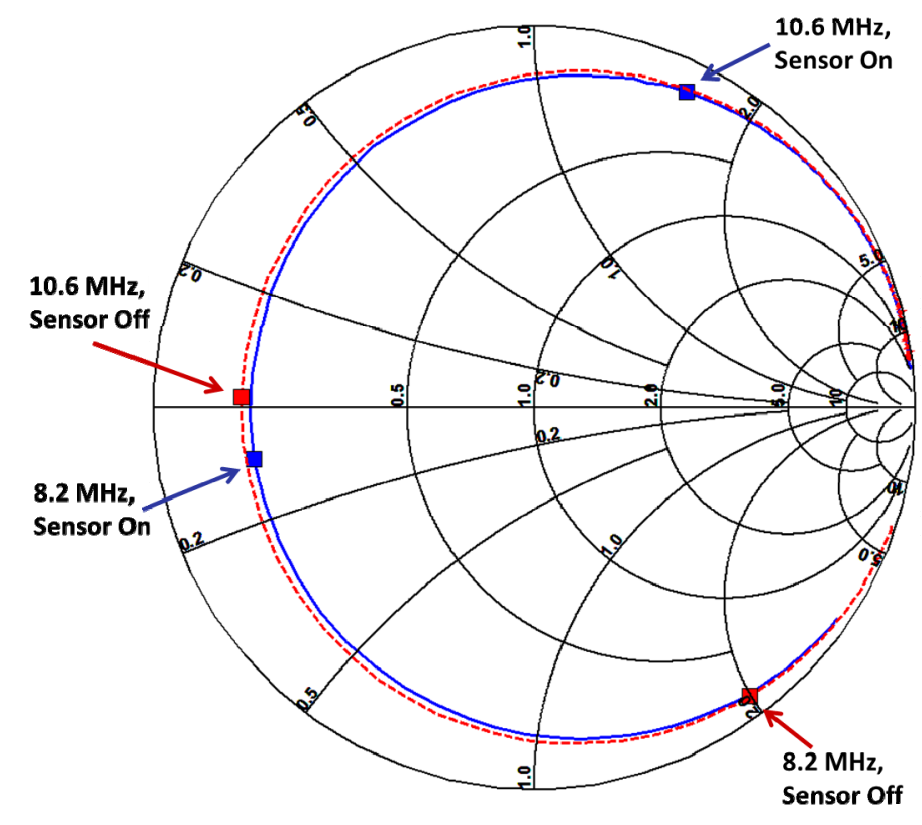

Impedance measurements are performed with a probe formed from a short stub of rigid coaxial cable with a metal outer jacket that has been extended with a $0.8 \mathrm{~mm}$ wire to create a two-point probe. That is, the coaxial assembly's center and outer conductor are used directly as measurement contact points. The rigid coaxial cable is connected with an SMA connector to a semi-rigid coaxial cable leading to the network analyzer. The procedure for impedance measurements with a balanced cable assembly and unbalanced device under test does provide room for improvements if further accuracy is demanded. One solution to decouple the tag from the transmission line, thereby suppressing current flow on the outer jacket of the cable assembly is to integrate a balun. Common approaches with using quarter wavelength baluns along the conductor is however problematic with $10 \mathrm{MHz}$ having a free space quarter wavelength of approximately $7.5 \mathrm{~m}$. Wavelength dependent devices are by definition also frequency dependent and thus not suitable for wideband measurements [33]. Another approach with potential for use in these kinds of measurements is to use a differential probe setup. For example where two stubs of the rigid coaxial cable have their outer metal shields soldered together and where the two ports of an antenna or chip are contacted by each center conductor. Transform equations for mixed-mode $S$-parameters as described in [34] and [35] can then be used to retrieve the desired differential mode $S$-parameters. Examples of using such measurement setup are found in [36] and [37]. For these relatively low frequencies a balun based on transformers and ferrite coils might also be used [33]. The results from measuring resonance peaks with a single-ended probe in this work however agree well with simulations and for triggering the EAS gates at 8.2 $\mathrm{MHz}$.

Figure 9 shows both simulated and measured transmission coefficients for the parallel plate tag (left) and the interdigital tag (right), where the values are normalized for each case. The measurements were performed on one tag of each type. It can be seen that the frequency shift for the "on" and "off" states are close to the simulated values. The difference between the simulated and measured values is 
attributed mostly to the manufacturing tolerance of the capacitances of the tags. Small variations in area and positioning of the screen printed silver paste that makes up the capacitors on the reverse side of the aluminum pattern, as seen in the right-hand portions of Figures 5 and 6, causes a small spread in the resonance frequency. Manufacturing tolerances are exemplified in Figure 10 and explains why the measured and simulated transmission coefficients in Figure 9 differ for the interdigital tag. To a smaller extent, the difference is also attributed to the added resistance caused by contacting the coils with silver paste, in the form of bridges for the parallel plate tag and as discrete contact points for the interdigital tag. The losses in the experimental data appears to be larger than for the simulated values as the graphs are wider for the experimental data and are attributed to the overall resistance of the tags as well as contact resistance of the probe when performing the measurements.

Figure 9. Simulated and measured transmission coefficients for parallel plate tag (left) and interdigital tag (right).
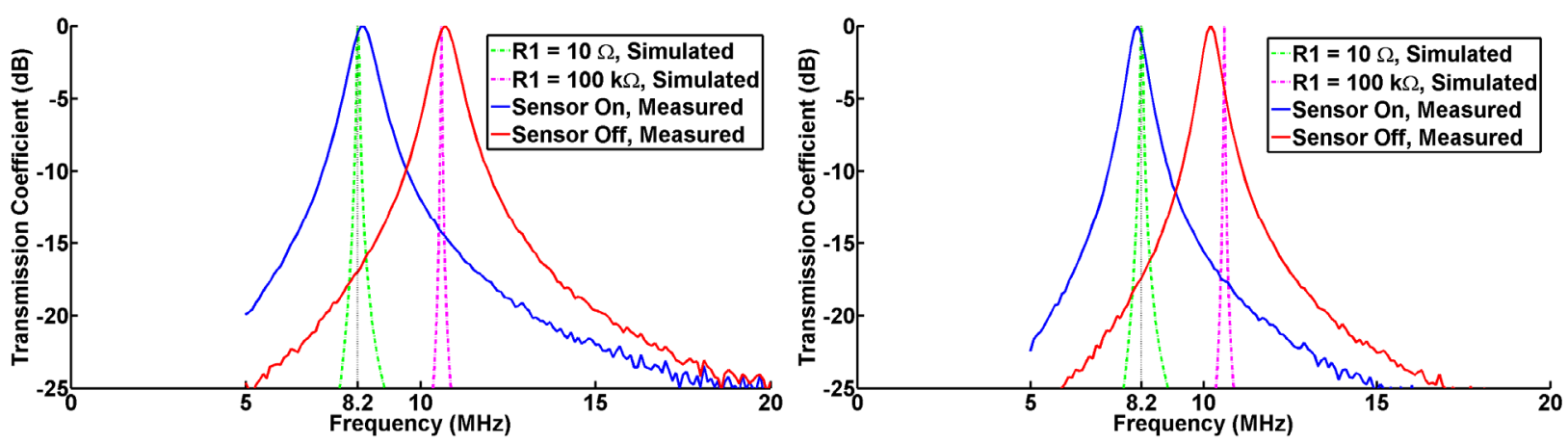

Figure 10. Plot (left) and histogram (right) showing the resonance frequency of 35 manufactured interdigital EAS tags as measured after the application of the screen print silver paste on the opposite side of the interdigital capacitor, without any adjustment of the capacitance.
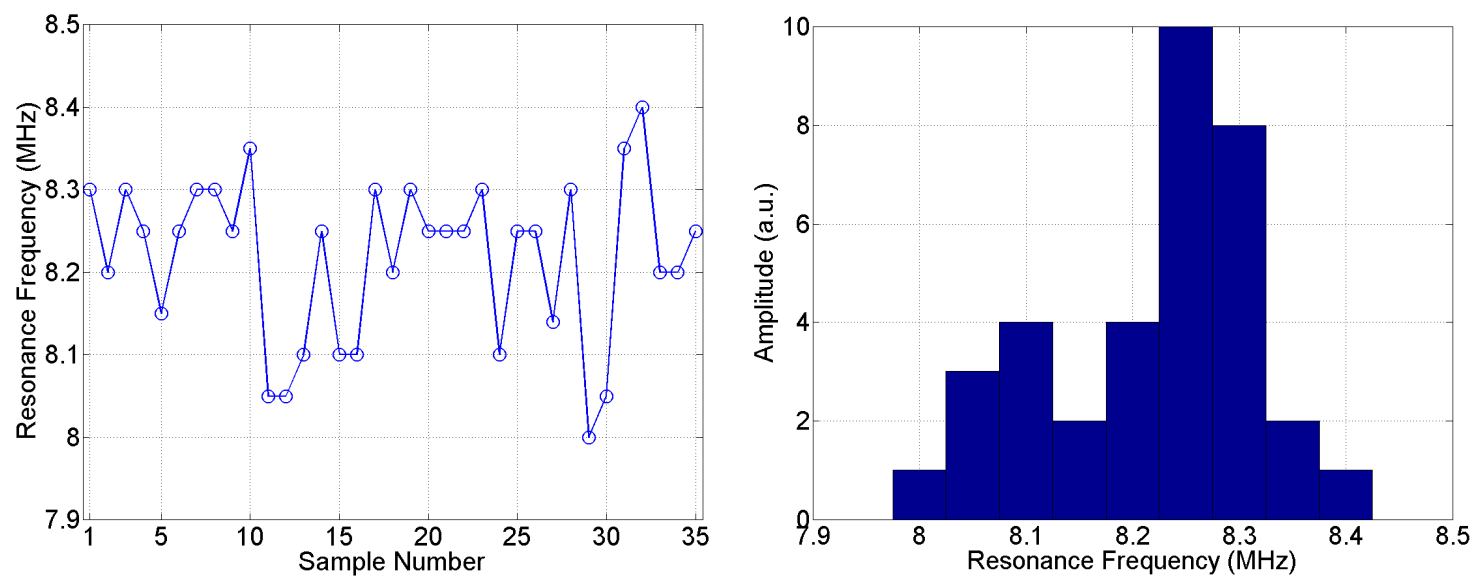

These frequencies are close to the predicted values obtained from the circuit simulations in the previous section.

The manufacturing of the tags appears to be robust, and from a series of 35 manufactured interdigital tags with screen printed silver paste and without adjusting them in any way, the average 
resonance frequency was measured to be $8.21 \mathrm{MHz}$ and the largest deviations were $\pm 0.2 \mathrm{MHz}$. A plot of the tags' resonance frequencies as well as a histogram of the same measurement is shown in Figure 10, where standard deviation is 0.097 .

The parallel plate type was not measured in this manner because the coil has to be cut to measure the resonant frequency, thereby destroying the tags from further use. However, of the tags measured, no larger deviation from the resonant frequency was observed than was the case for the interdigital tags.

The possibility to adjust the capacitance by the application of screen printed silver paste after manufacturing is also a possibility.

When considering using sensor equipped EAS tags in applications, it is of interest to discuss the possible reading methods and read ranges. Standard EAS tag readers used in shops are positioned as gates at the entrance, detecting tags that pass through. Since the tags are 1-bit devices there is no option of distinguishing between different tags. The distance between the gates is usually a few meters. One possible scenario when using the modified sensor EAS tags is to let goods pass through standard EAS reader gates that are for example positioned so that they enclose a conveyor belt. It is also a possibility that the tags are read when positioned in front of a reader system rather than passing through gates, for example with a handheld reader.

Tests were performed by positioning reader gates (manufactured by Gunnebo Gateway, Sweden) side by side and reading commercial standard EAS tags in front of those. See Figure 11 for the setup used.

Figure 11. EAS detection gate setup where the gates have been turned side by side to test the reading range of EAS tags positioned in front of the gates.

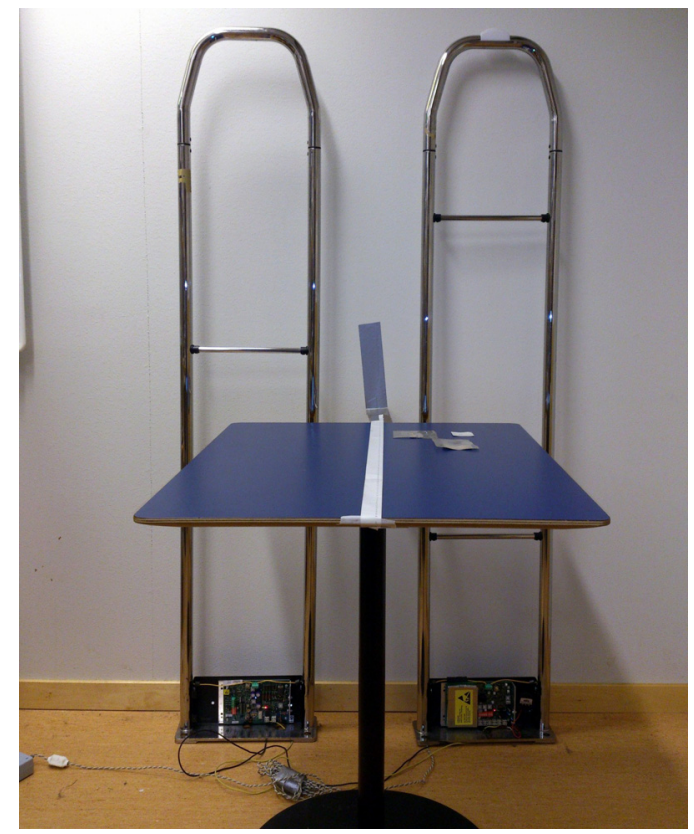

In this case the tags can be read at a distance of about $30 \mathrm{~cm}$. This can be viewed as a best case scenario: any additional resistance added will make the maximum read distance less. In this article is shown a concept of using sensors together with a modified EAS tag and the total resistance of the demonstrated experimental tags will be larger than if such tags were manufactured commercially. This 
is because of added contact resistance when mounting the sensors and the screen printed part of the tags, as can be seen in Figure 5. The modified sensor tags are only readable at about $5 \mathrm{~cm}$ in its current form, but will approach the commercial tags if its serial resistance is decreased.

In the conventional use of EAS tags it is important to avoid false alarms or tags passing close to but not through the gates, and therefore there is really no interest in having a too long read range or to sensitive reading.

To be able to detect the modified EAS tags at the two different resonance frequencies a modification to the existing equipment is required. As the system is designed now it sweeps a few $100 \mathrm{KHz}$ below and above 8.2 MHz. Detection at several frequencies will lead to added complexity and some extra cost of the readers, but from a technical perspective to read in the range $8-10 \mathrm{MHz}$ should be straightforward. Other issues include radiation regulatory issues with more wideband EAS gates, although in the ETSI EN $300330-1$ standard, the $5 \mathrm{kHz}-30 \mathrm{MHz}$ range is permitted for use in short range inductive devices [38].

A handheld reader would also be operated at a short distance where the tags are positioned within $0-1 \mathrm{~m}$ to avoid crosstalk between several nearby tags.

If longer read range or more functionality is needed it is probably a better option to move to an RFID solution that, at a larger cost, could provide more functionality and longer read range. Such a solution could be of different types, such as a battery operated commercial solution as well as some of the options presented in research [15-17]. The difference in cost can however be too large for some cost sensitive areas that can be satisfied with the performance offered by the more limited read range and non-identifiable tags of the EAS system. The cost of manufacturing standard EAS tags is very low and the technology can be said to be both mature and having a very high production volume because it is used in almost all shops, attached to even the cheapest goods. Therefore, it is possible to argue that this is the cheapest tag with regards to material and production cost of any conceivable RF or RFID tag in use today. If considering modification of a standard EAS tag to consist of an LCC circuit, which should be easily done by splitting the capacitor into two capacitors during production. It is reasonable to say that the added cost will be the sensor and its attachment and not the modification. A reasonable assumption is thus that the cost of the sensor and its attachment is equivalent if the sensor is used with a modified EAS tag or an RFID tag. The cost difference will then be the cost for the RFID tag compared to the EAS tag.

When considering the manufacturing cost of EAS tags and RFID tags, commercial or research, the cost for the RFID chip and its chip attachment procedure will always be added to the cost of the actual antenna and this will be higher than the EAS tag, which does not have any chip.

\section{Conclusions}

The manufactured modified LCCR type EAS tags have been shown to work as a sensor platform for resistive sensors. The demonstrated case, with a tilt sensor, is merely an example of a possible sensor application. The types of sensors that are possible for use in this case are limited to resistive sensors, which have low resistance when the EAS tag triggers the readout, preferably only a few ohms. Tags of such a type can be used with standard EAS tag readout systems thus making it possible to build a system of commercially available standard components. It is also shown that the deviation in 
resonance frequency of the manufactured tags is low, as shown in Figure 10, but still visible as a small difference between the simulated and measured transmission coefficients in Figure 9. Disadvantages with EAS technologies as compared to chip-based RFID solutions include the absence of anti-collision methods. It is thus very difficult to separate the signal from two or more EAS tags in the same reading zone, putting constraints of the maximum density of EAS tags. The span of available sensors might also be partly narrower for EAS systems, since it is known that a lower sensor resistance is needed to operate an EAS tag than for integrating sensors to UHF RFID tags. Until fully printed RFID tags are realized, the cost in manufacturing EAS sensor tags does not have to consider the cost of silicon chips. The applications can vary, but the main target is generally those applications where a large number of tags with sensors are necessary and the price sensitivity is so high that more advanced RFID tags prove to be too expensive.

\section{References}

1. Subramanian, V.; Frechet, J.M.J.; Chang, P.C.; Huang, D.C.; Lee, J.B.; Molesa, S.E.; Murphy, A.R.; Redinger, D.R.; Volkman, S.K. Progress Toward Development of All-Printed RFID Tags: Materials, Processes, and Devices. Proc. IEEE 2005, 93, 1330-1338.

2. Subramanian, V.; Chang, P.C.; Lee, J.B.; Molesa, S.E.; Volkman, S.K. Printed organic transistors for ultra-low-cost RFID applications. IEEE Trans. Components Packag. Technol. 2005, 28, 742-747.

3. Allen, M.L.; Jaakkola, K.; Nummila, K.; Seppa, H. Applicability of Metallic Nanoparticle Inks in RFID Applications. IEEE Trans. Components Packag. Technol. 2009, 32, 325-332.

4. Redinger, D.; Molesa, S.; Yin, S.; Farschi, R.; Subramanian, V. An Ink-Jet-Deposited Passive Component Process for RFID. IEEE Trans. Elec. Dev. 2004, 51, 1978-1983.

5. Rida, A.; Vyas, R.; Basat, S.; Bhattacharya, S.K.; Tentzeris, M.M. Novel Manufacturing Processes for Ultra-Low-Cost Paper-Based RFID Tags with Enhanced "Wireless Intelligence". In Proceedings of 57th Electronic Components and Technology, Reno, NV, USA, May 2007; pp. 773-776.

6. Vyas, R.; Rida, A.; Tentzeris, M.M. Design and Development of a Novel Paper-based Inkjet-Printed RFID-Enabled UHF (433.9 MHz). In Proceedings of the Sensor Node Asia-Pacific Microwave Conference, Bangkok, Thailand, December 2007; pp. 1-4.

7. Virtanen, J.; Björninen, T.; Ukkonen, L.; Kaija, K.; Joutsenoja, T.; Sydänheimo, L.; Elsherbeni, A.Z. The Effect of Conductor Thickness in Passive Inkjet Printed RFID Tags. In Proceedings of Antennas and Propagation Society International Symposium, Toronto, Canada, July 2010; pp. 1-4.

8. Sidén, J.; Nilsson, H-E. Line Width Limitations of Flexographic-Screen- and Inkjet printed RFID Antennas. In Proceedings of Antennas and Propagation Society International Symposium, Honolulu, HI, USA, 2007; pp. 1745-1748.

9. Rida, A.; Yang, L.; Vyas, R.; Tentzeris, M.M. Conductive Inkjet-Printed Antennas on Flexible Low-Cost Paper-Based Substrates for RFID and WSN Applications. IEEE Antennas Propag. Mag. 2009, 51, 13-23. 
10. Vyas, R.; Lakafosis, V.; Rida, A.; Chaisilwattana, N.; Travis, S.; Pan, J.; Tentzeris, M.M. Paper-Based RFID-Enabled Wireless Platforms for Sensing Applications. IEEE Trans. Microw. Theory Tech., 2009, 57, 1370-1382.

11. Yang, L.; Zhang, R.; Staiculescu, D.; Wong, C.P.; Tentzeris, M.M. A Novel Conformal RFID-Enabled Module Utilizing Inkjet-Printed Antennas and Carbon Nanotubes for Gas-Detection Applications. IEEE Antennas Wirel. Propag. Lett. 2009, 8, 653-656.

12. Siegel, A.C.; Phillips, S.T.; Dickey, M.D.; Lu, N.; Suo, Z.; Whitesides, G.M. Foldable Printed Circuit Boards on Paper Substrates. Adv. Funct. Mater. 2010, 20, 28-35.

13. Osvaldo, J.; de la Jarrige, E.L.; Boone, F. Creating screen-printed passive components for microwave applications. Microelectron. Int. 2010, 27, 84-92.

14. Sidén, J.; Nilsson, H-E. Line width limitations of flexographic-screen- and inkjet printed RFID antennas. In Proceedings of IEEE Antennas and Propagation Society International Symposium, Honolulu, HI, USA, 2007; pp. 1745-1748.

15. Marrocco, G.; Amato, F. Self-sensing passive RFID: From theory to tag design and experimentation. In Proceedings of IEEE Microwave Conference, Rome, Italy, September 2009; pp. 1-4.

16. Philipose, M.; Smith, J.R.; Jiang, B.; Mamishev, A.; Sumit, R.; Sundara-Rajan, K. Battery-free wireless identification and sensing. Pervasive Comput. 2005, 4, 37-45.

17. Caizzone, S.; Occhiuzzi, C.; Marrocco, G. Multi-Chip RFID Antenna Integrating Shape-Memory Alloys for Detection of Thermal Thresholds. IEEE Trans. Antennas Propag. 2011, 59, 2488-2494.

18. Finkenzeller, K. RFID Handbook, 2nd ed.; Wiley: Chichester, England, UK, 2003; pp. 32-33.

19. SCA Cool Logistics International Group Home page. Available online: http://www.sca-coollogistics.com (accessed on 02 November 2012).

20. Pacquit, A.; Frisby, J.; Diamond, D.; Lau, K.T.; Farrell, A.; Quilty, B.; Diamond, D. Development of a smart packaging for the monitoring of fish spoilage. Food Chem. 2007, 102, 466-470.

21. Mahalik, N.P.; Nambiar, A.N. Trends in food packaging and manufacturing systems and technology. Trends Food Sci. Technol. 2010, 21, 117-128.

22. Tan, E.L.; Ng, W.N.; Shao, R.; Pereles, B.D.; Ong, K.G. A wireless, passive sensor for quantifying packaged food quality. Sensors 2007, 7, 1747-1756.

23. Stojanović, G.; Radovanović, M.; Malešev, M.; Radonjanin, V. Monitoring of Water Content in Building Materials Using a Wireless Passive Sensor. Sensors 2010, 10, 4270-4280.

24. Curranoa, J.; Yub, M.; Balachandran, B. Latching in a MEMS shock sensor: Modeling and experiments. Sensors Actuators A: Phys. 2010, 159, 41-50.

25. Lim, N.; Kim, J.; Lee, S.; Kim, N.; Cho, G. Screen Printed Resonant Tags for Electronic Article Surveillance Tags. IEEE Trans. Adv. Packag. 2009, 32, 72-76.

26. Öhlund, T.; Örtegren, J.; Andersson, H.; Nilsson, H-E. Sintering Methods for Metal Nanoparticle Inks on Flexible Substrates. In Proceedings of 25th International Conference on Digital Printing Technologies/Digital Fabrication, Louisville, KY, USA, 2009; pp. 614-617.

27. Yung, K.C.; Gu, X.; Lee, C.P.; Choy, H.S. Ink-jet printing and camera flash sintering of silver tracks on different substrates. J. Mater. Process. Technol. 2010, 210, 2268-2272.

28. Allen, M.L.; Aronniemi, M.; Mattila, T.; Alastalo, A.; Ojanpera, K.; Suhonen, M.; Seppa, H. Electrical sintering of nanoparticle structures. Nanotechnology 2008, 19, 175201. 
29. Greer, J.R.; Street, R.A. Thermal cure effects on electrical performance of nanoparticle silver inks. Acta Mater. 2007, 55, 6345-6349.

30. Perelaer, J.; de Laat, A.W.M.; Hendriks, C.E.; Schubert, U.S. Inkjet-printed silver tracks: Low temperature curing and thermal stability investigation. J. Mater. Chem. 2008, 18, 3209-3215.

31. Magdassi, S.; Grouchko, M.; Berezin, O.; Kamyshny, A. Triggering the Sintering of Silver Nanoparticles at Room Temperature. ACS Nano 2010, 4, 1943-1948.

32. Zapka, W.; Voit, W.; Loderer, C.; Lang, P. Low temperature chemical post-treatment of inkjet printed nano-particle silver inks. In Proceedings of 24th International Conference on Digital Printing Technologies/Digital Fabrication, Pittsburgh, PA, USA, 2008; pp. 906-911.

33. Balanis, C.A. Antenna Theory Analysis and Design; Harper \& Row: New York, NY, USA, 1982, pp. 480-483.

34. Bockelman, D.E.; Eisenstadt, W.R. Combined Differential and Common-Mode Scattering Parameters: Theory and Simulation. IEEE Trans. Microw. Theory Tech. 1995, 43, 1530-1539.

35. Bockelman, D.E.; Eisenstadt, W.R. Pure-mode network analyzer for on-wafer measurements of mixed-mode $S$-parameters of differential circuits. IEEE Trans. Microw. Theory Tech. 1997, 45, 1071-1077.

36. Qing, X.; Goh, C.K.; Chen, Z.N. Measurement of UHF RFID tag antenna impedance. In Proceedings of IEEE International Workshop on Antenna Technology, Santa Monica, CA, USA, 2009; pp. 1-4.

37. Zhu, H.; Ko, Y.C.A.; Ye, T.T. Impedance measurement for balanced UHF RFID tag antennas. In Proceedings of IEEE Radio and Wireless Symp, New Orleans, LA, USA, 2010; pp. 128-131.

38. ETSI Homepage. Available online: http://etsi.org/WebSite/homepage.aspx (accessed on 10 October 2012).

(C) 2012 by the authors; licensee MDPI, Basel, Switzerland. This article is an open access article distributed under the terms and conditions of the Creative Commons Attribution license (http://creativecommons.org/licenses/by/3.0/). 